\title{
Propiedades Morfológicas en la Parte Media de la Díafisis del Hueso Metacarpiano III de Equino Mestizo Criollo
}

\author{
Morphological Properties in the Mid Part of the Metacarpal \\ III Bone Diaphysis of Equine Criollo Crossbreed
}

Moine, R."; Galán, A.*; Vivas, A."; Fioretti, C.*; Varela, M."; Bonino, F.**; Quinteros, R.** \& Natali, J."

MOINE, R.; GALÁN, A.; VIVAS, A.; FIORETTI, C.; VARELA, M.; BONINO, F.; QUINTEROS, R. \& NATALI, J. Propiedades morfológicas en la parte media de la díafisis del hueso metacarpiano III de equino mestizo criollo. Int. J. Morphol., 33(3):955-961, 2015.

RESUMEN: El desarrollo del esqueleto es de máxima importancia en caballos de trabajo y alta competencia. Los miembros torácicos sostienen el $60 \%$ del peso corporal y están sometidos a esfuerzos biomecánicos que los predisponen a lesiones, estando obligados a especializarse en el apoyo. El metacarpiano III soporta la mayor carga entre los huesos del cuerpo y la sustancia compacta tiene gran espesor dorsal y medial. Es un material complejo que posee plasticidad y fuerza considerable, con capacidad para soportar la deformación y recuperar su forma. El objetivo de este estudio es aportar conocimientos morfológicos, aplicables a la clínica animal, del hueso metacarpiano III de equino mestizo criollo. Se estudiaron los huesos metacarpiano III de 30 equinos hembras y machos, entre 18 meses y 5 años de edad, divididos en dos grupos etarios, provenientes del frigorífico General Pico, Las Higueras. Se determinó: peso y longitud total; diámetro latero-medial y dorso-palmar en la parte media de la diáfisis. Al metacarpiano III izquierdo se le practicó una osteotomía transversal en la parte media de la diáfisis para determinar: espesor de la cortical, área cortical, área medular y área total. Los datos fueron analizados mediante técnicas de correlación y regresión lineal simple, análisis de la varianza y comparaciones múltiples de Tukey usando el paquete estadístico InfoStat, con un nivel de significación de 0,05 . Los resultados muestran que existe asociación entre el peso versus área cortical $(\mathrm{r}=0,76, \mathrm{p}=0,0001)$ y longitud del hueso versus área cortical $(\mathrm{r}=0,74 \mathrm{p}=0,0001)$, el área depende linealmente del peso y longitud del hueso ( $\mathrm{R} 2=0,97, \mathrm{p}<0,0001$ y $\mathrm{R} 2=0,96, \mathrm{p}<0,0001$ respectivamente). Se comprobó que existen diferencias significativas entre los diámetros de las áreas y espesor de los cuadrantes ( $\mathrm{p}<0,0001$ ), al contrastar los cuadrantes entre sí se observaron diferencias a excepción del cuadrante dorsal y lateral. Existe diferencias entre las áreas de los distintos grupos etários (p=0,0034). La morfología tiene influencia sobre las propiedades biomecánicas del hueso.

PALABRAS CLAVE: Equino; Hueso metacarpiano III; Morfometría.

\section{INTRODUCCIÓN}

El desarrollo del sistema esquelético es un importante requerimiento para un caballo con potencial de competición y trabajo (Bigot et al., 1996). En el caballo, los miembros torácicos sostienen en mayor medida el peso corporal, ya que el $60 \%$ del peso se encuentra apoyado en ellos debido a la posición craneal del centro de gravedad y en consecuencia están sometidos a un mayor esfuerzo biomecánico. El metacarpiano III soporta la mayor carga entre los huesos del cuerpo (Bartel et al., 1978). La sustancia compacta (cortical) es de gran espesor en sus cuadrantes dorsal y medial y responde como un material complejo que posee plasticidad y fuerza considerable con capacidad para soportar deformación y recuperación de su forma, además cumple funciones mecánicas y protectoras. El metacarpiano III del caballo posee poca cobertura de tejidos blandos, excepto en su cara palmar (Richardson, 2006) y posee alta susceptibilidad a sufrir lesiones (Aguiar et al., 2012).

En el hueso metacarpiano III de caballo, las fuerzas pasan a lo largo del hueso (Turner et al., 1998). La cortical del hueso, absorbe de distinta manera las cargas: el cuadrante dorsal y medial soportan más carga de tensión, mientras que el cuadrante lateral y palmar soportan más cargas de compresión, en el cuadrante lateral influye la acción del músculo extensor digital lateral (Bigot et al.). Las áreas corticales del hueso son indicadores de formación y resorción del teji-

\footnotetext{
* Anatomía Animal, Dpto. de Anatomía Animal. Facultad de Agronomía y Veterinaria. Universidad Nacional de Río Cuarto, Río Cuarto, Argentina. ** Matemática y Bioestadística. Dpto. de Ciencias Básicas y Agropecuarias. Facultad de Agronomía y Veterinaria. Universidad Nacional de Río Cuarto, Río Cuarto, Argentina.

Este trabajo fue subsidiado por Scyt-UNRC (A322)
} 
do óseo durante el crecimiento (Nunamaker et al., 1991). El tejido óseo esta influenciado por dos procesos generales: morfológicos (cambios en el tamaño y forma) y remodelación ósea (cambios en la arquitectura interna). Estos cambios se encuentran predominantemente en caballos jóvenes y con menor frecuencia en caballos adultos (Stover et al., 1992).

Autores como Schryver (1978), Hanson et al. (1995); Bigot et al., Reilly \& Burnstein (1975) y Nunamaker et al. (1989) investigaron sobre las propiedades morfológicas y mecánicas del hueso metacarpiano III de caballo. El Shorafa et al. (1979), Hanson \& Markel (1994) y Lawrence et al. (1994) en sus trabajos con hueso metacarpiano III de caballo relacionaron área cortical con contenido mineral y edad del animal. Moine et al. (2001) estudiaron la morfometría del metacarpiano III en yeguas mestizas criollas, mientras que Galán et al. (2004) estudiaron las medidas geométricas del metacarpiano III en potrillos mestizo criollo.

El presente trabajo tiene el propósito de brindar informaciones aplicables a la clínica animal sobre las propiedades morfológicas del hueso metacarpiano III de caballo mestizo criollo y se fundamenta en la importancia clínica y quirúrgica de la mano del caballo. El objetivo fue evaluar la morfología del hueso metacarpiano III de caballo mestizo criollo en la parte media de la diáfisis del hueso.

\section{MATERIAL Y MÉTODO}

Se estudiaron los huesos metacarpianos III, provenientes de 30 equinos (hembras y machos) jóvenes mestizos-criollos con edades que oscilaron entre 18 meses y 5 años y que no presentaron problemas de aplomo. Se dividió al conjunto de animales en dos grupos etarios: G1 (18 meses a 2 años) y G2 (2 a 5 años), la edad se determinó por cronometría dentaria. Las muestras se obtuvieron del frigorífico General Pico, Las Higueras. Una vez extraídos los huesos metacarpianos III se liberaron de los tejidos blandos por disección convencional.

Los huesos metacarpianos III izquierdos se pesaron con balanza (2600 g de tara), no se extrajeron los huesos metacarpianos II y IV. Se midió la longitud con regla milimétrica (escala $300 \mathrm{~mm}$ ) desde la cresta dorso-palmar a proximal hasta la cresta sagital distal del hueso. En la parte media de la diáfisis de los huesos metacarpianos III izquierdo (Fig. 1), se midió el diámetro latero-medial y dorso-palmar y en el mismo lugar se seccionó transversalmente al hueso. En la superficie de sección del hueso se midió el espesor de la cortical en sus cuadrantes: dorsal, palmar, medial y lateral y se evaluó área total, área cortical y área de la cavidad medular (Fig. 1), se usó para las mediciones un calibrador milimétrico (escala $0,02 \mathrm{~mm}$ ).

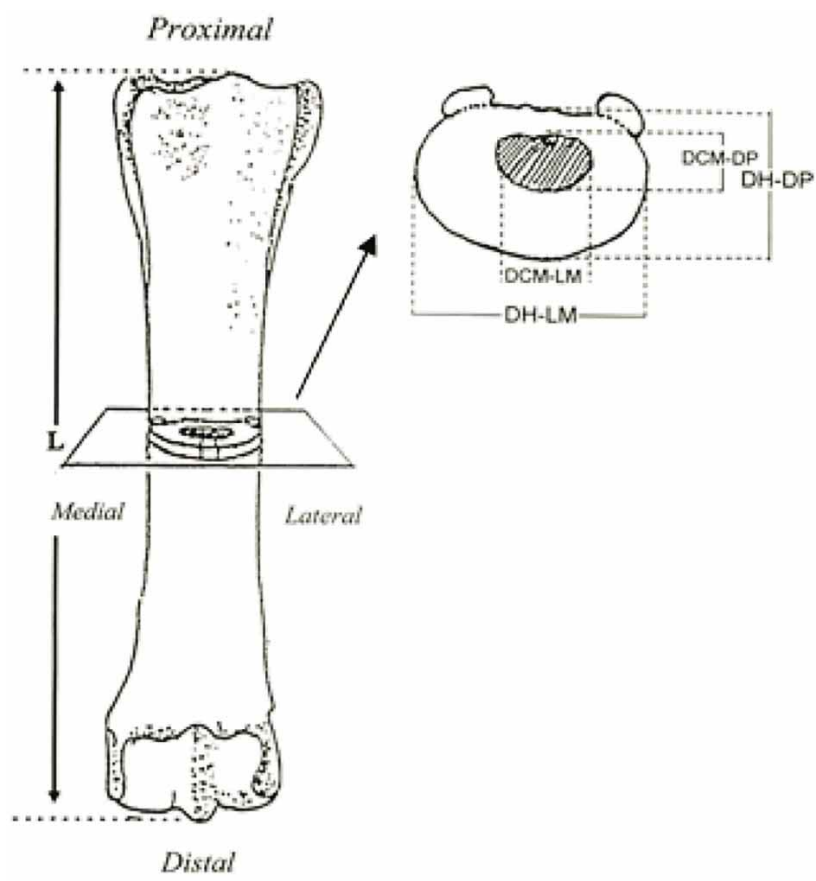

Fig. 1. Hueso metacarpiano III seccionado en la parte media de la diáfisis y superficie de sección: DH-DP= diámetro del hueso dorso-palmar; DH-LM= diámetro del hueso latero-medial; DCM-DP= diámetro cavidad medular dorso-palmar; DCM-LM= diámetro cavidad medular latero-medial.

Los datos fueron analizados mediante el uso de la técnica de correlación y regresión lineal simple, análisis de la varianza y comparaciones múltiples de Tukey usando el paquete estadístico InfoStat (2009) con un nivel de significación de 0,05 . Para las variables cuantitativas: de peso, longitud, diámetros y espesor, se realizó una tabla con los valores muestrales de tendencia central y de dispersión. Para las variables tipos de áreas, sexo, espesor de los cuadrantes y grupos etarios se construyeron Diagramas de caja.

La estadística inferencial empleada fueron las pruebas de correlación lineal simple (para las variables peso versus área cortical, longitud versus área cortical) y análisis de regresión lineal simple. También se efectuó análisis de la varianza para la variable espesor de cuadrantes y Prueba de Tukey con un Nivel de significación $=0,05$.

\section{RESULTADOS}

Las medidas de tendencia central de cada variable tomadas del metacarpiano III izquierdo se muestran en la Tabla I. 
MOINE, R.; GALÁN, A.; VIVAS, A.; FIORETTI, C.; VARELA, M.; BONINO, F.; QUINTEROS, R. \& NATALI, J. Propiedades morfológicas en la parte media de la díafisis del hueso metacarpiano III de equino mestizo criollo. Int. J. Morphol., 33(3):955-961, 2015.

Tabla I. Medidas resumen del hueso metacarpiano III izquierdo $(\mathrm{n}=30)$.

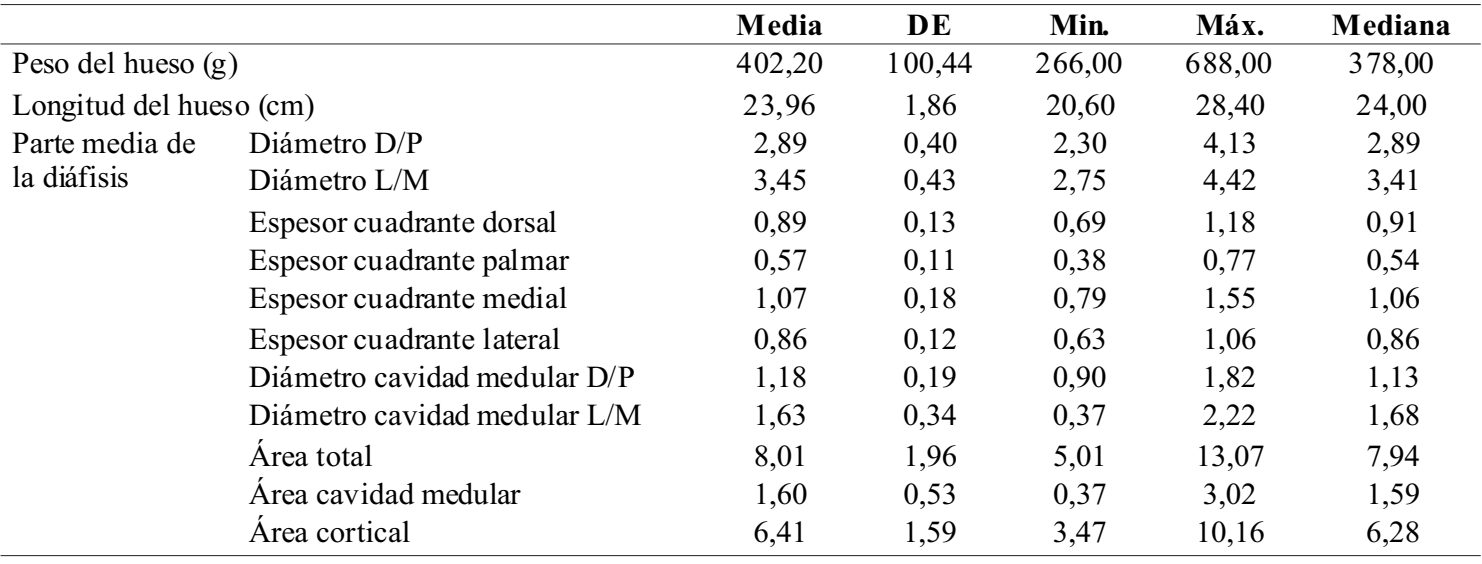

$\mathrm{D} / \mathrm{P}=$ dorso-palmar; $\mathrm{L} / \mathrm{M}=$ latero-medial.

La Figura 2 muestra Diagrama de caja para área total, área de la cavidad medular y área cortical en la parte media de la diáfisis del metacarpiano III izquierdo, se observan que existen diferencias entre las áreas.

La Figura 3 muestra Diagrama de caja para área cortical versus sexo, el cual muestra que hay diferencias de áreas entre machos y hembras.

La Figura 4 muestra Diagrama de caja para los cuadrantes dorsal, medial, lateral y palmar en la parte media de la diáfisis del metacarpiano III, se observa que existen diferencias en el espesor entre los distintos cuadrantes. El Diagrama de caja para área cortical versus grupos etarios muestra que hay diferencias de área cortical entre los distintos grupos (Fig. 5).

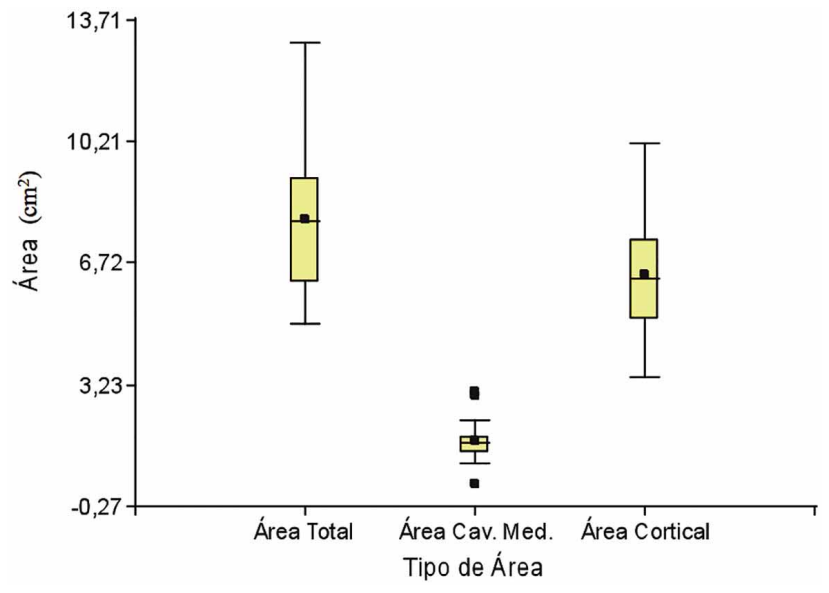

Fig. 2. Diagrama de caja para las áreas total, cavidad medular y cortical en la parte media de la diáfisis del metacarpiano III izquierdo $(n=30)$.

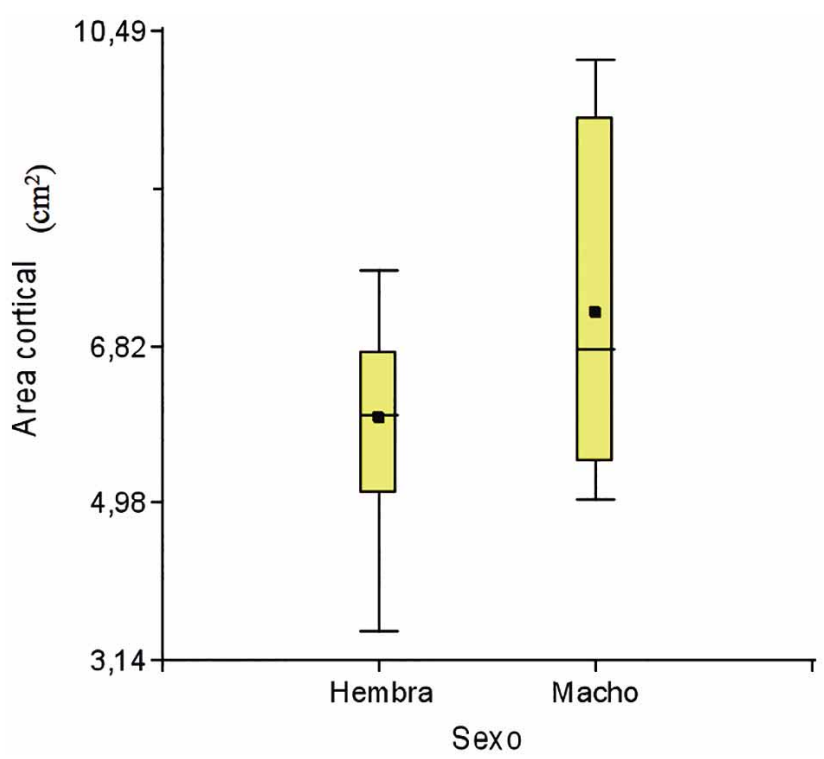

Fig. 3. Diagrama de caja para área cortical vs. sexo $(n=30)$.

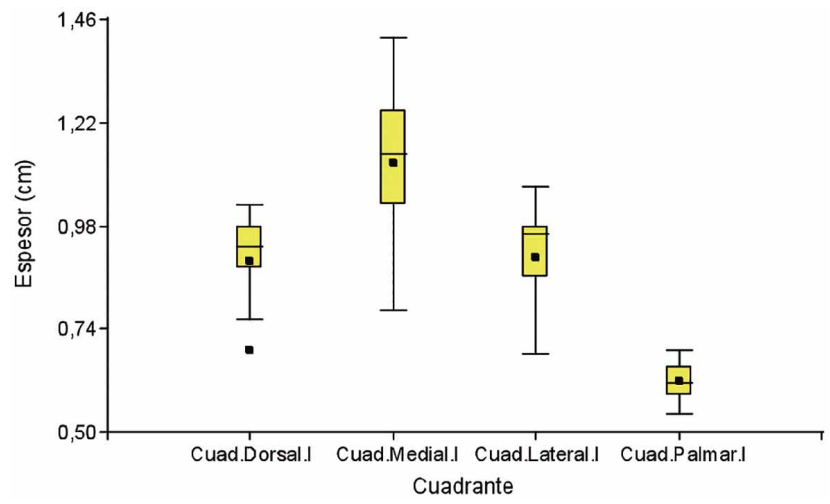

Fig. 4. Diagrama de caja para los cuadrantes dorsal, medial, lateral y palmar en la parte media de la diáfisis del metacarpiano III izquierdo $(n=30)$. 
En la Tabla II se muestra el análisis de correlación para las variables peso versus área cortical $(\mathrm{r}=0,76$, $\mathrm{p}<0,0001)$, se observa la asociación entre las dos variables. La Tabla III muestra el coeficiente de determinación del área cortical versus peso $\left(R \sum=0,97\right)$, se observa en la Tabla IV el coeficiente de regresión $(p<0,0001)$ y estadísticos asociados para la variable peso. La recta de regresión se muestra en la Figura 6, la cual representa que el área cortical depende linealmente del peso del hueso.

Tabla II. Correlación de Pearson: coeficientes\probabilidades para las variables peso vs. área cortical.

\begin{tabular}{lcc}
\hline & Peso $(\mathbf{g})$ & Área cortical $\left(\mathbf{c m}^{2}\right)$ \\
\hline Peso $(\mathrm{g})$ & 1,00 & $3,3 \mathrm{E}-06$ \\
Área cortical $\left(\mathrm{cm}^{2}\right)$ & 0,76 & 1,00 \\
\hline
\end{tabular}

Tabla III. Análisis de regresión para las variables área cortical vs. peso. Coeficiente de determinación.

\begin{tabular}{lcc}
\hline Variable & $\mathbf{n}$ & $\mathbf{R}_{-}$ \\
\hline Área cortical $\left(\mathrm{cm}^{2}\right)$ & 30 & 0,97 \\
\hline
\end{tabular}

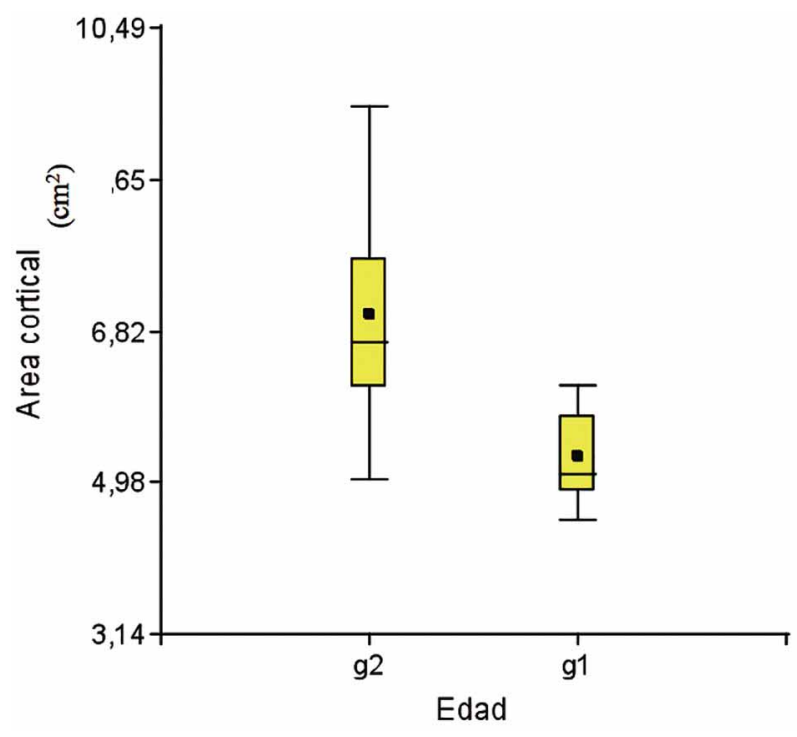

Fig. 5. Diagrama de caja para área cortical vs. edad $(n=30)$.

Tabla IV. Coeficientes de regresión y estadísticos asociados para la variable peso.

\begin{tabular}{lcccccc}
\hline Coef & Est. & E.E. & LI(95\%) & LS(95\%) & T & p-valor \\
\hline Peso $(\mathrm{g})$ & 0,02 & $5,1 \mathrm{E}-04$ & 0,01 & 0,02 & 30,99 & $<0,0001$ \\
\hline
\end{tabular}

La Tabla $\mathrm{V}$ muestra el análisis de correlación para las variables longitud versus área cortical $(r=0,74$, $\mathrm{p}<0,0001)$, se observa en ella la asociación entre las dos variables. En la Tabla VI se muestra el coeficiente de determinación $\left(R \sum=0,96\right)$ para área cortical vs. longitud del hueso. En la Tabla VII se presentan los coeficientes de regresión $(\mathrm{p}<0,0001)$ y estadísticos asociados para la variable longitud. La recta de regresión se muestra en la Figura 7, en la cual se observa que el área cortical depende linealmente de la longitud del hueso.

Tabla V. Correlación de Pearson: coeficientesłprobabilidades para las variables longitud vs. área cortical.

\begin{tabular}{lcc}
\hline & Longitud $(\mathbf{c m})$ & Área cortical $\left(\mathbf{c m}^{2}\right)$ \\
\hline Longitud $(\mathrm{cm})$ & 1,00 & $7,0 \mathrm{E}-06$ \\
Área cortical $\left(\mathrm{cm}^{2}\right)$ & 0,74 & 1,00 \\
\hline
\end{tabular}

Tabla VI. Análisis de regresión para las variables área cortical vs. longitud. Coeficiente de determinación.

\begin{tabular}{lcc}
\hline Variable & n & $\mathbf{R}_{-}$ \\
\hline Área cortical $\left(\mathrm{cm}^{2}\right)$ & 30 & 0,96 \\
\hline
\end{tabular}

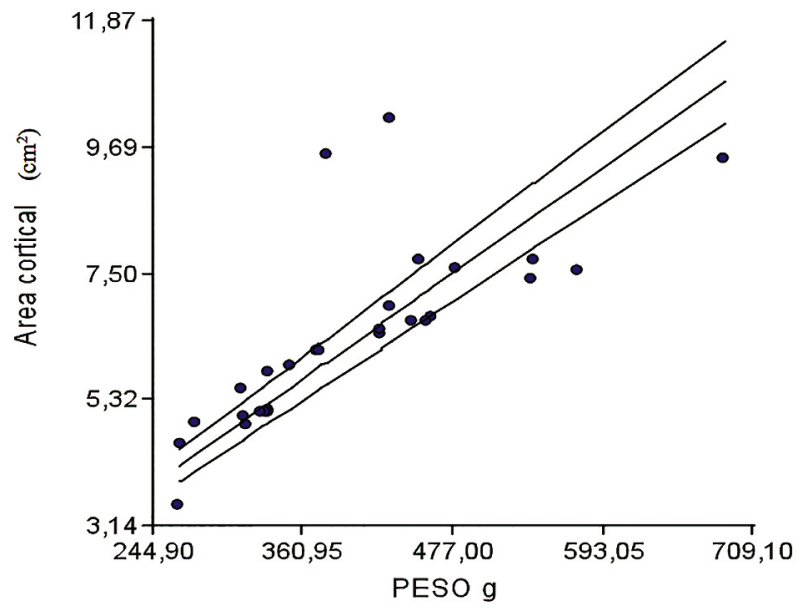

Fig. 6. Regresión lineal simple para la variable área cortical vs. peso $(n=30)$. 
MOINE, R.; GALÁN, A.; VIVAS, A.; FIORETTI, C.; VARELA, M.; BONINO, F.; QUINTEROS, R. \& NATALI, J. Propiedades morfológicas en la parte media de la díafisis del hueso metacarpiano III de equino mestizo criollo. Int. J. Morphol., 33(3):955-961, 2015.

Tabla VII. Coeficientes de regresión y estadísticos asociados para la variable longitud.

\begin{tabular}{lcccccc}
\hline Coef. & Est. & E.E. & LI(95\%) & LS(95\%) & T & p-valor \\
\hline Longitud $(\mathrm{cm})$ & 0,27 & 0,01 & 0,25 & 0,29 & 27,07 & $<0,0001$ \\
\hline
\end{tabular}

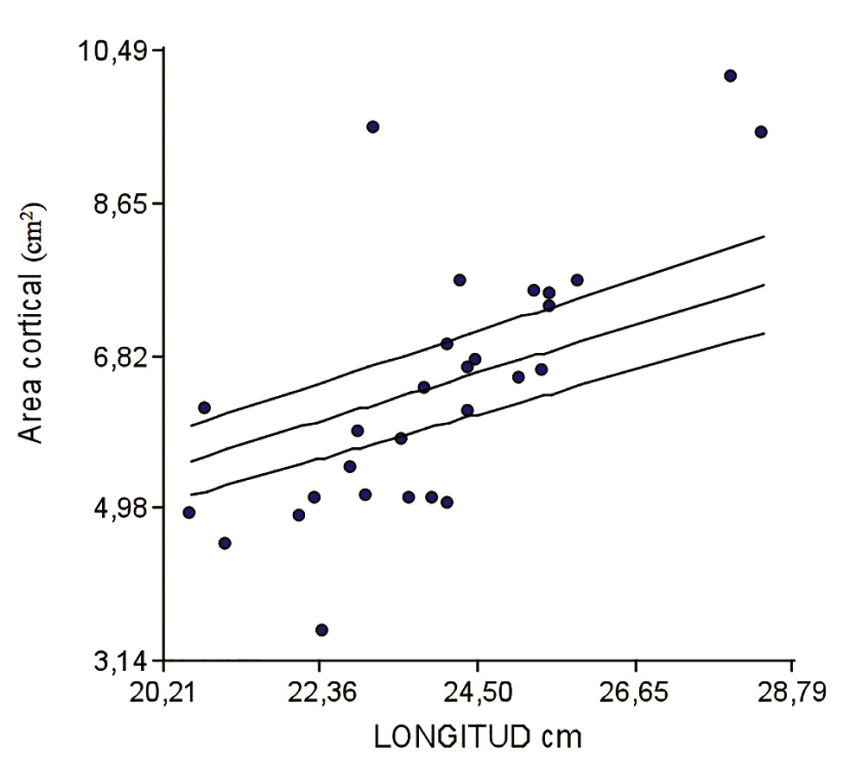

Fig. 7. Regresión lineal simple para la variable área cortical vs. longitud $(n=30)$.

En la Tabla VIII se muestra el análisis de la varianza para el espesor de los cuadrantes y la Tabla IX muestra el contraste entre los cuadrantes, se observa que hay contraste entre ellos a excepción del cuadrante dorsal con el cuadrante lateral $(\mathrm{p}<0,05)$.

\section{DISCUSIÓN}

Las propiedades morfológicas del metacarpiano III del caballo están íntimamente relacionadas con las propiedades biomecánicas y estructurales del hueso. El hueso contrarresta las cargas mediante adaptaciones en su geometría que involucra una actividad continua y coordinada de construcción y destrucción (Currey, 1984).

Los resultados de este trabajo muestran que el peso promedio del metacarpiano III izquierdo fue de $402,20 \pm 100,44 \mathrm{~g}$, valor inferior a lo observado por Moine et al. en yeguas mestizas criollas $(427,20 \pm 48,50 \mathrm{~g})$ y superior a lo encontrado por Galán et al. en potrillos mestizos criollos $(383,20 \pm 52,45 \mathrm{~g})$.
Tabla VIII. Análisis de la varianza para la variable espesor.

\begin{tabular}{lccccc}
\hline F.V. & SC & gl & CM & F & p-valor \\
\hline Modelo & 3,56 & 3 & 1,19 & 63,75 & $<0,0001$ \\
Posición & 3,56 & 3 & 1,19 & 63,75 & $<0,0001$ \\
Error & 2,01 & 108 & 0,02 & --- & --- \\
Total & 5,58 & 111 & --- & --- & --- \\
\hline
\end{tabular}

Tabla IX. Prueba de Tukey para la comparación de cuadrantes. Nivel de significación $=0,05$.

\begin{tabular}{lcccc}
\hline & 1 Dorsal & 2 Lateral & 3 Medial & 4 Palmar \\
\hline 1 Dorsal & --- & --- & $*$ & $*$ \\
2 Lateral & sd & --- & $*$ & $*$ \\
3 Medial & sd & sd & --- & $*$ \\
4 Palmar & sd & sd & sd & --- \\
$*=$ indican diferencias significativas para el nivel elegido. &
\end{tabular}

El valor promedio de longitud del hueso metacarpiano III izquierdo encontrado en este estudio es de $23,96 \pm 1,86$ $\mathrm{cm}$, inferior a los valores encontrados por Moine et al. en yeguas mestizas criollas $(24,07 \pm 0,79 \mathrm{~cm})$ y por Hanson \& Markel en caballos $(26,8 \pm 0,22 \mathrm{~cm})$. Nuestros resultados son similar a los observados por Galán et al., en potrillos mestizos criollos cuyos valores reportados fueron de $23,89 \pm 1,14$ $\mathrm{cm}$.

Este trabajo muestra que el diámetro dorso-palmar promedio en la parte media de la diáfisis del hueso metacarpiano III izquierdo es de $2,89 \pm 0,40 \mathrm{~cm}$, siendo estos valores similares a los encontrados por Moine et al., quien publicó valores de 2,72 $\pm 0,17 \mathrm{~cm}$ en sus trabajos en yegua. Hanson et al. publicaron valores promedios de 3,0 a 3, $1 \pm 0,06$ $\mathrm{cm}$ en sus estudios en caballos. Galán et al. reportaron valores promedios de 2,60 $\pm 0,13 \mathrm{~cm}$ para huesos metacarpianos III de potrillos mestizos criollos. La medida latero-medial promedio del hueso en la parte media de la diáfisis del metacarpiano III es de $3,45 \pm 0,43 \mathrm{~cm}$, inferior a lo encontrado por Moine $e t$ al., en yeguas cuyo valores fueron 3,65 $\pm 0,18$ $\mathrm{cm}$. Galán et al. en sus estudios en potrillos mestizos criollos, encuentra valores similares a los nuestros. Se observa 
que el promedio del diámetro dorso-palmar del hueso es menor que el diámetro latero-medial, lo cual manifiesta la forma característica del hueso quien estaría ligado a su biomecánica. Los diámetros del hueso están asociados a su forma, es así como se diseñan para neutralizar las tensiones por efecto de la flexión resultante de la carga y las fuerzas opuestas generadas por la masa muscular (Gupta \& Zioupos, 2008).

En este trabajo, las medidas dorso-palmar y lateromedial promedio de la cavidad medular en la parte media de la diáfisis del hueso metacarpiano III izquierdo fueron de $1,18 \pm 0,19 \mathrm{~cm}$ y $1,63 \pm 0,34 \mathrm{~cm}$ respectivamente. Estos datos son similares a los observados por Moine et al. en metacarpiano III de yegua y por Galán et al. en metacarpiano III de potrillo mestizo criollo.

Considerando el espesor de la cortical tomada en la parte media de la diáfisis del metacarpiano III izquierdo, se observa que el cuadrante medial presenta un espesor mayor $(1,07 \pm 0,18 \mathrm{~cm})$ que los otros tres cuadrantes. Este valor coincide con lo publicado por Hanson et al., Moine et al. y Galán et al., quienes hicieron sus estudios en equinos de diferentes razas y edades, yeguas mestizas criollas y potrillos mestizos criollos respectivamente. Los valores promedios del espesor de la cortical de los otros tres cuadrantes: dorsal, lateral y palmar fueron de $0,89 \pm 0,13 \mathrm{~cm}, 0,86 \pm 0,12 \mathrm{~cm}$ y $0,57 \pm 0,11$ $\mathrm{cm}$, respectivamente. Se observa en dichos valores que el cuadrante palmar presenta el menor espesor cortical.

Existen diferencias estadísticamente significativas entre el espesor promedio de los cuadrantes con un $\mathrm{p}<0,0001$. El test de comparaciones múltiple de Tukey da un nivel de significación del 0,05 donde las diferencias están entre los cuadrantes dorsal y medial; dorsal y palmar; lateral y medial; lateral y palmar; medial y palmar, no observándose diferencias entre los cuadrantes lateral y dorsal.

La diferencia en el espesor de los cuadrantes estaría relacionada con la intensidad de la carga, el tendón del músculo extensor digital lateral tiene efecto sobre el cuadrante lateral y el tendón del músculo extensor digital común tienen efecto sobre el cuadrante dorsal (Bigot et al.).

En este trabajo los valores promedios observados en la superficie de sección de la mitad de la diáfisis del hueso metacarpiano III fueron: $8,01 \pm 1,96 \mathrm{~cm}^{2}, 6,41 \pm 1,59 \mathrm{~cm}^{2}$ y $1,60 \pm 0,53 \mathrm{~cm}^{2}$ para área total, área cortical y área medular respectivamente. Ellos no se diferencian de los publicados por Moine et al. en su estudio en yeguas, en cambio Galán et al. en su estudio en potrillo mestizo criollo, publicaron valores inferiores para las tres áreas, 7,18 $\pm 0,77 \mathrm{~cm}^{2}$; $5,74 \pm 0,55 \mathrm{~cm}^{2}$ y $1,42 \pm 0,37 \mathrm{~cm}^{2}$ para área total, cortical y medular respectivamente.
Existe asociación entre el peso del hueso versus área cortical $(r=0,76, p=0,0001)$ y longitud del hueso versus área cortical $(\mathrm{r}=0,74, \mathrm{p}=0,0001)$. Se infiere entonces, que el área depende linealmente del peso y longitud del hueso $\left(\mathrm{R} \sum=0,97, \mathrm{p}<0,0001\right.$ y $\mathrm{R} \sum=0,96, \mathrm{p}<0,0001$ respectivamente). Existe diferencias entre las áreas de los distintos grupos etarios $(p=0,0034)$.

Durante los primeros 5 años de vida hay un incremento de área total, dicho proceso es acompañado por un incremento de área cortical mayor que el incremento que se produce en el área medular. Coincide lo anterior con Nunamaker et al. (1989) quien encontró que el área cortical aumenta claramente entre el primer y quinto año de edad al igual que el crecimiento del área total.

En conclusión decimos que: el diámetro latero-medial en la parte media de la diáfisis del metacarpiano III izquierdo de caballo mestizo criollo es mayor que el diámetro dorso-palmar. El espesor del cuadrante medial en la parte media de la diáfisis del metacarpiano III izquierdo presenta una superficie mayor que los otros tres cuadrantes siendo el cuadrante palmar el de menor tamaño. El tamaño del área cortical depende linealmente del peso y longitud del hueso. La intensidad de la carga a la que está expuesto el hueso condiciona el crecimiento del espesor de la cortical. En los primeros 5 años de vida hay un crecimiento mayor del área cortical que el crecimiento simultáneamente producido en el de área medular.

AGRADECIMIENTOS. Frigorífico General Pico, Las Higueras, por proveer las muestras.

MOINE, R.; GALÁN, A.; VIVAS, A.; FIORETTI, C.; VARELA, M.; BONINO, F.; QUINTEROS, R. \& NATALI, J. Morphological properties in the mid part of the metacarpal III bone diaphysis of equine criollo crossbreed. Int. J. Morphol., 33(3):955$961,2015$.

SUMMARY: The development of high skeleton is important in working horses and associated high competition. Thoracic members hold $60 \%$ of body weight and are subjected to biomechanical efforts that predispose the animal to injury, being forced to rely on the support. The large metacarpal III supports load between bones of the body and the compact substance is large dorsal and medial thickness. It is composed of complex material and possesses plasticity and considerable force, with the ability to sustain deformation and subsequently recover its form. The objective of this study is to provide morphometric knowledge applied to animal clinic of the metacarpal III bone of equine criollo crossbreed. Thirty (30) metacarpal III bones of male and female horses, between 18 months and 5 years old, divided into two age groups, from the Frigorifico General Pico, Las Higueras groups 
were studied. We determined: weight, total length, diameter lateromedial and dorsal-palmar in the middle of the diaphysis. A transverse osteotomy was performed left to metacarpal III in the middle diaphysis to determine: cortical thickness, cortical area, medullary area and total area. The data was analyzed by correlation and simple linear regression techniques, analysis of variance and Tukey multiple comparisons using the statistical InfoStat (2009) package, with a significance level of 0.05 . The results showed an association between weight vs. cortical area $(\mathrm{r}=0.76, \mathrm{p}=0.0001)$ and bone length vs. cortical area $(\mathrm{r}=0.74, \mathrm{p}=0.0001)$, the area depends linearly on the weight and length bone $(\mathrm{R} 2=0.97, \mathrm{p}<0.0001$ and $\mathrm{R} 2=0.96, \mathrm{p}<0.0001$ respectively). It was found that there are significant differences between the diameters of the area and thickness of the quadrants $(\mathrm{p}<0.0001)$, contrasting quadrants were observed in the dorsal and lateral exception quadrant. There are differences between areas of different age groups $(p=0.0034)$. The morphology influences the biomechanical properties of the bone.

KEY WORDS: Equine; Metacarpal III bone; Morphometric.

\section{REFERENCIAS BIBLIOGRÁFICAS}

Aguiar, A. C. S.; Machado, V. M. V.; Santos, R. V.; Puoli Filho, J. N. P.; Mota, M. D. S. \& Vulcano, L. C. Estudo da correlação da densitometria óssea e do perímetro diafisário medial do terceiro metacarpiano em equinos. Arq. Bras. Med. Vet. Zootec., 64(4):1065-9, 2012.

Bartel, D. L.; Schryver, H. F.; Lowe, J. E. \& Parker, R. A. Locomotion in the horse: a procedure for computing the internal forces in the digit. Am. J. Vet. Res., 39(11):1721-7, 1978.

Bigot, G.; Boudizi, A.; Rumelhart, C. \& Martin-Rosset, W. Evolution during growth of the mechanical properties of the cortical bone in equine cannon-bones. Med. Eng. Phys., 18(1):79-87, 1996.

Currey, J. D. The mechanical properties of materials and the estructure of bone. In: Currey, J. D. (Ed.). The mechanical Adaptation of Bone. New Jersey, Princeton University Press, 1984. pp.3-37.

El Shorafa, W. M.; Feaster, J. P. \& Ott, E. A. Horse metacarpal bone: age, ash content, cortical area and failure stress interrelationships. J. Anim. Sci., 49(4):979-82, 1979.

Galán, A. M.; Rivera, M. C.; Moine, R.; Ferraris, G.; Gigena, M. S. \& Natali, J. Propiedades morfométricas del metacarpiano III de potrillos mestizos. Rev. Chil. Anat., 20(3):285-90, 2004.

Gupta, H. S. \& Zioupos, P. Fracture of bone tissue: The 'hows' and the 'whys'. Med. Eng. Phys., 30(10):1209-26, 2008.

Hanson, P. D. \& Markel, M. D. Radiographic geometric variation of equine long bones. Am. J. Vet. Res., 55(9):1220-7, 1994.
Hanson, P. D.; Markel. M. D. \& Vanderby, R. Jr. Diaphyseal structural properties of equine long bones. Am. J. Vet. Res., 56(2):233-40, 1995.

Lawrence, L. A.; Ott, E. A.; Miller, G. J.; Poulos, P. W.; Piotrowski, G. \& Asquith, R. L. The mechanical properties of equine third metacarpals as affected by age. J. Anim. Sci., 72(10):2617-23, 1994.

Moine, R. M.; Rivera, M. C.; Vivas, A. B.; Ferraris, G. R.; Galán, A. M. \& Natali, J. Morfometría y determinación de calcio y fósforo en la parte media de la diáfisis del metacarpiano III en yeguas mestiza con criollo. Arch. Med. Vet., 33(1):63-8, 2001.

Nunamaker, D. M.; Butterwerck, D. M. \& Provost, M. T. Some geometric properties of the third metacarpal bone: a comparison between the thoroughbred and standardbred racehorse. $J$. Biomech., 22(2):129-34, 1989.

Nunamaker, D. M.; Butterwerck, D. M. \& Black, J. In vitro comparison of Thoroughbred and Standardbred racehorses with regard to local fatigue failure of the third metacarpal bone. Am. J. Vet. Res., 52(1):97-100, 1991.

Reilly, D. T. \& Burnstein, A. H. The elastic and ultimate properties of compact bone tissue. J. Biomech., 8(6):393-405, 1975.

Richardson, D. W. Metacarpal and Metatarsal Bones. In: Auer, J. A. \& Stick, J. A. Equine Surgery. $3^{\text {a }}$ ed. Philadelphia, Elsevier, 2006. pp.1238-53.

Schryver, H. F. Bending properties of cortical bone of the horse. Am. J. Vet. Res., 39(1):25-8, 1978.

Stover, S. M.; Pool, R. R.; Martin, R. B. \& Morgan, J. P. Histological features of the dorsal cortex of the third metacarpal bone middiaphysis during postnatal growth in thoroughbred horses. $J$. Anat., 181(Pt. 3):455-69, 1992.

Turner, C. H. Three rules for bone adaptation to mechanical stimuli. Bone, 23(5):399-407, 1998.

\section{Dirección para Correspondencia: \\ José Natali \\ Anatomía Animal, \\ Departamento de Anatomía Animal \\ Facultad de Agronomía y Veterinaria \\ Universidad Nacional de Río Cuarto \\ Campus Universitario (5800) \\ Rio Cuarto, Cba \\ ARGENTINA}

Email: jnatali@ayv.unrc.edu.ar
Recibido : 01-11-2014

Aceptado: 28-05-2015 